\title{
Giant outburst of EXO 2030+375 observed with INTEGRAL: pulse resolved spectral analysis
}

\author{
D. Klochkov ${ }^{* a}$, A. Santangelo ${ }^{a}$, R. Staubert ${ }^{a}$, C. Ferrigno ${ }^{b}$ \\ ${ }^{a}$ Institut für Astronomie und Astrophysik, Universität Tübingen (IAAT) \\ ${ }^{b}$ INAF IFC-Pa, via U. La Malfa 153, 90146 Palermo, Italy \\ E-mail: klochkoveastro.uni-tuebingen.de
}

In June-September 2006 Be/X-ray binary EXO 2030+375 experienced the second since its discovery giant outburst. The source was shown to have a complicated pulse averaged X-ray spectral continuum with possible evidences of cyclotron absorption features. In our contribution we present for the first time the analysis of pulse-resolved broad-band X-ray spectra of EXO 2030+375 during a giant outburst. Our analysis includes the data obtained with the INTEGRAL observatory close to the maximum and during the decay phase of the 2006 giant outburst. We report strong variability of the spectrum with pulse phase. Possible evidence of an absorption line around $64 \mathrm{keV}$ at pulse phase preceeding the main peak of the pulse profile is present in the spectra obtained close to the maximum of the outburst. The variation of spectral fit parameters with pulse phase is discussed. Alternative spectral models are compared.

7th INTEGRAL Workshop

September 8-11 2008

Copenhagen, Denmark

\footnotetext{
${ }^{*}$ Speaker.
} 


\section{Introduction}

The transient accreting pulsar EXO 2030+375 is part of a high mass X-ray binary system with a B0 Ve optical companion. The orbital period and eccentricity are $\sim 46 \mathrm{~d}$ and $\sim 0.42$, respectively. The system belongs to the class of Be/X-ray binaries which is the most numerous population of massive X-ray binaries in the Galaxy. Such systems typically show two types of outburst behavior:

(1) Normal (or type I) outbursts. They are characterized by relatively low X-ray luminosities $L_{\mathrm{X}} \sim 10^{36}-10^{37} \mathrm{erg} \mathrm{s}^{-1}$ and low spin-up rates (if any), and reccurence at the orbital period (or its multiples). Such outbursts last for days to weeks and are associated with the periastron passages of the neutron star.

(2) Giant (or type II) outbursts. They are characterized by higher X-ray luminosities $L_{X} \gtrsim$ $10^{37} \mathrm{erg} \mathrm{s}^{-1}$ and high spin-up rates. Such outbursts occur irregularly, they last several weeks and are not correlated with any particular orbital phase. The typical recurrence time is of the order of several years. Giant outbursts are thought to be due to a dramatic expansion of the disk surrounding the Be star, leading to the formation of an accretion disk around the compact object (Coe 2000; Ziolkowski 2002).

There were two giant outbursts in the history of observations of EXO 2030+375. During the first one the source was discovered by EXOSAT in 1985 (Parmar et al. 1989b). The second outburst took place in June-September 2006 and has been observed by INTEGRAL, Swift and RXTE. A preliminary pulse averaged spectral analysis of some of these observations was presented in Klochkov et al. (2007). A detailed analysis of pulse averaged RXTE spectra obtained during the outburst was performed by Wilson et al. (2008). It has been shown that the pulse averaged $\mathrm{X}$-ray continuum of the source has a complicated shape and cannot be modelled by a simple power law/cutoff model. Wilson et al. (2008) included an absorption line at $\sim 10 \mathrm{keV}$ (which they interpreted as a cyclotron line) in their spectral model whereas Klochkov et al. (2007) have shown that the spectrum can be equally well fitted without the absorption line, but including a broad emission "bump" at $\sim 15 \mathrm{keV}$.

Here we present for the first time pulse-phase resolved broad band (3-150 keV) spectra of EXO 2030+375 during a giant outburst. For our analysis we use all available INTEGRAL data taken during the 2006 outburst. The X-ray continuum of the source shows strong variability with pulse phase, with some features being present only at particular pulse phase intervals.

\section{Observations}

The INTErnational Gamma Ray Astrophysics Laboratory (INTEGRAL, Winkler et al. 2003) performed three pointed observations of EXO 2030+375 during its giant outburst in June-September 2006. Two observations (on 19-20 August and 23-25 September) were done close to the maximum of the outburst. The third one (on 6-8 October) was performed at the end of the decay phase, when the X-ray luminosity dropped by a factor of $\sim 10$ with respect to the maximum of the outburst. A part of the RXTE/ASM light curve ${ }^{1}$ including the giant outburst is shown in Fig. 1. The times of the INTEGRAL observations are indicated and referred to as Set 1, Set 2, and Set 3 throughout the paper.

\footnotetext{
${ }^{1}$ We used the results provided by the ASM/RXTE team.
} 


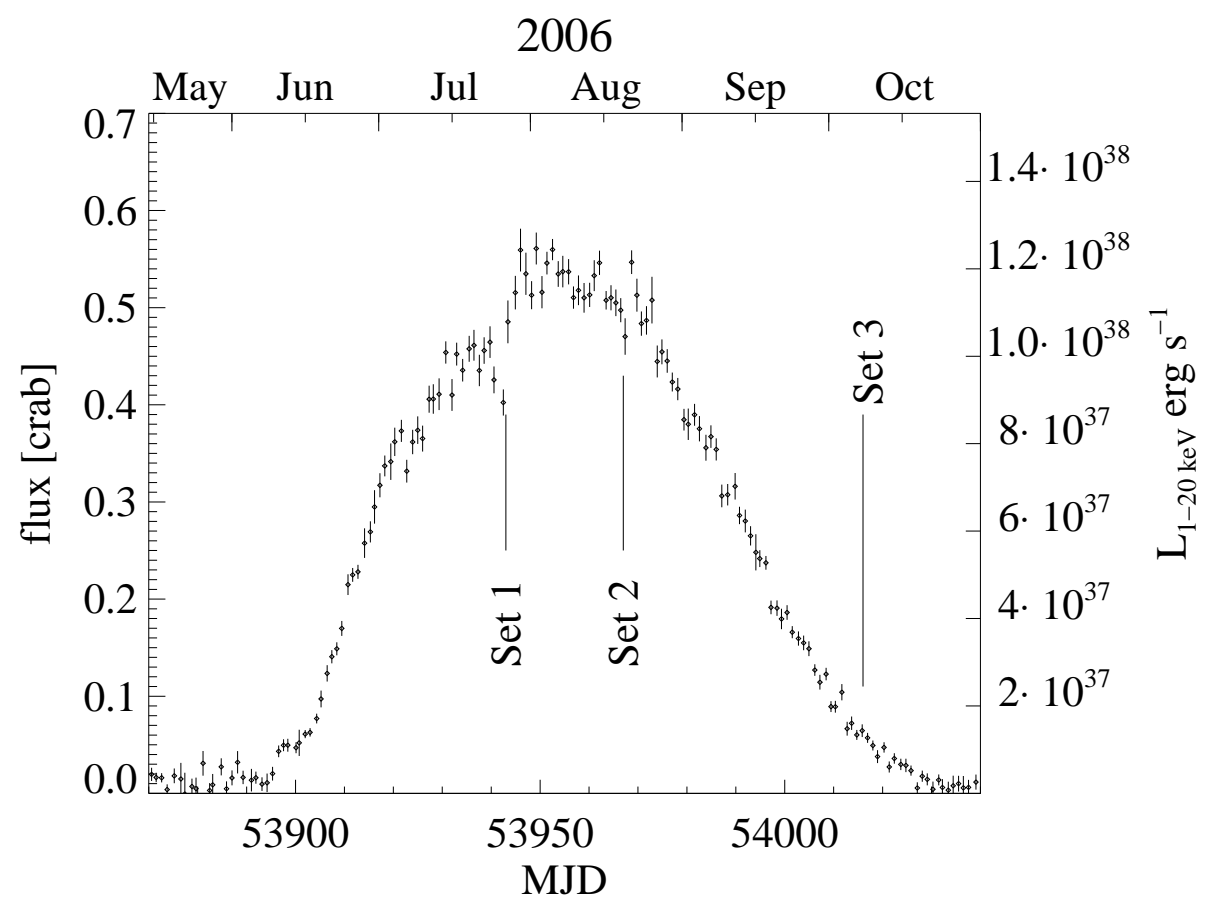

Figure 1: The RXTE/ASM light curve of EXO 2030+375 showing the 2006 giant outburst. Times of INTEGRAL observations analyzed in this work are marked with vertical lines. The observations are referred to as Set 1, Set 2, and Set 3 in the text.

Table 1: The pulse periods and associated derivatives for three sets of observations analyzed in this work. They were used to construct pulse profiles and to define the pulse phase for the pulse-resolved analysis. The uncertainties in parentheses $(68 \%)$ refer to the last digit(s).

\begin{tabular}{cccc}
\hline \hline Observation & $\begin{array}{c}\text { Reference } \\
\text { epoch (MJD) }\end{array}$ & $\begin{array}{c}P \\
{[\mathrm{~s}]}\end{array}$ & $\begin{array}{c}-d P / d t \\
{\left[10^{-8} \mathrm{~s} / \mathrm{s}\right]}\end{array}$ \\
\hline & & & \\
Set 1 & 53942.240864 & $41.58084(3)$ & $2.96(21)$ \\
Set 2 & 53966.392169 & $41.519270(5)$ & $2.758(20)$ \\
Set 3 & 54014.010200 & $41.45425(6)$ & $0.22(23)$ \\
\hline
\end{tabular}

Observations corresponding to Sets 1 and 2 were performed close to the maximum of the outburst (Fig. 1). During Set 1 which is roughly half as long as Set 2 the main target of observations was Cyg X-3 located $4^{\circ}$ away from EXO 2030+375. Therefore, the statistics of the EXO 2030+375 data obtained during Set 1 is noticeably worse than that during Set 2. As a result, our pulse phase resolved analysis of the maximum of the outburst is mainly driven by the data taken in Set 2 .

\section{Energy-resolved pulse profiles}

Figure 2 presents energy-resolved background-subtracted pulse profiles of the source obtained during the observations corresponding to Set 2 . The corresponding pulse period and its derivative as well as the zero epoch are provided in Table 1. The pulse profiles in the energy bands below 

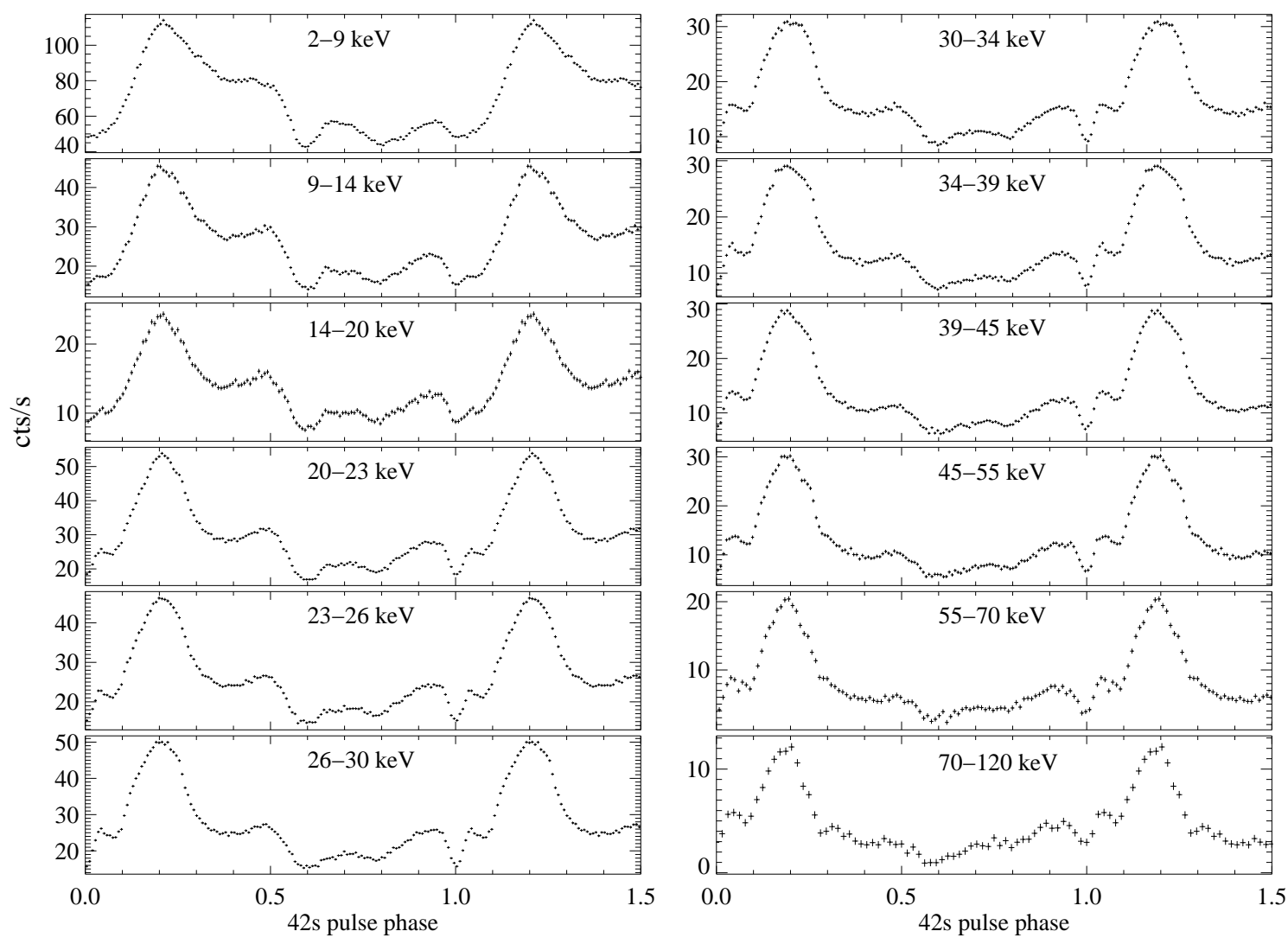

Figure 2: Energy resolved pulse profiles of EXO 2030+375 obtained with JEM-X (below $20 \mathrm{keV}$ ) and IBIS/ISGRI (above $20 \mathrm{keV}$ ) in Set 2 (where the data have the best statistics).

$20 \mathrm{keV}$ were obtained from the JEM-X data, while for energies above $20 \mathrm{keV}$ the IBIS/ISGRI data were used. As one can see, the shape of the profile changes smoothly with energy indicating variations of the $\mathrm{X}$-ray spectrum with pulse phase.

\section{Pulse-resolved spectra}

We have performed a separate analysis of the spectra accumulated in different pulse phase intervals. Phase binning was chosen to provide similar statistics of spectra in each bin and to have better phase resolution around the main peak where the most rapid spectral changes are expected. Figure 3 shows unfolded pulse phase resolved spectra of the source (they are shifted vertically with respect to each other in order to avoid overlaps). Variability of the spectral continuum is clearly seen.

As it was pointed in Sect. 1, the pulse-averaged spectral continuum of the source is rather complicated and cannot be modelled by any of the simple spectral functions (a power law modified at higher energies by an exponential cutoff) which are usually used to fit spectra of accreting pulsars. In an attempt to model the spectrum Wilson et al. (2008) modified a power law/cutoff model by a Gaussian absorption line at $\sim 10 \mathrm{keV}$ while Klochkov et al. (2007) included a broad Gaussian emission component at $\sim 15 \mathrm{keV}$ instead. In this work we tried to use both models to fit 


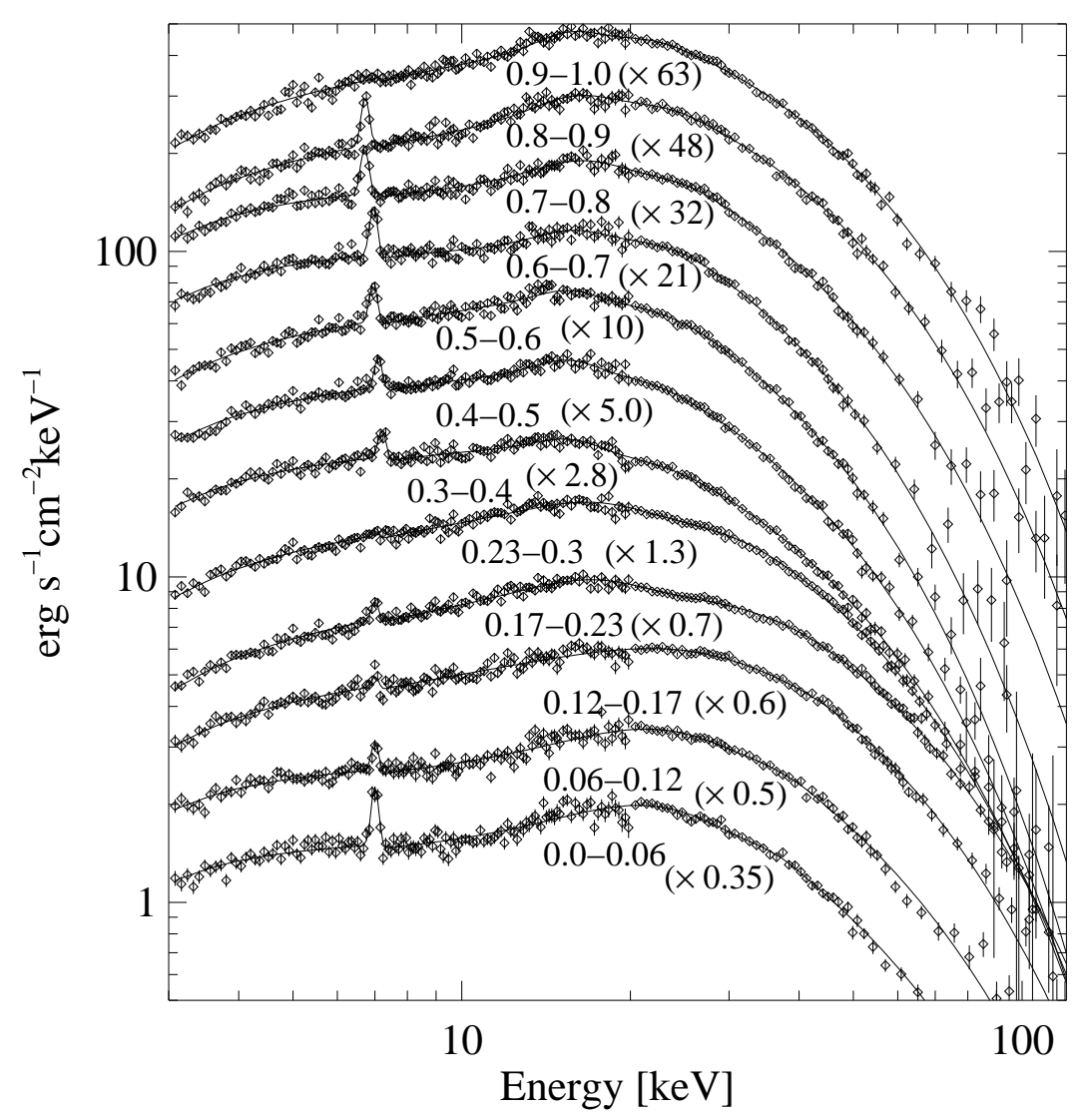

Figure 3: Pulse-resolved broad band X-ray spectra of EXO 2030+375 obtained in Set 2. Phase bins are indicated (phase zero is the same as in Fig. 2. Values in parentheses indicate the multiplicative factor applied to the flux in each spectrum to avoid overlaps. The solid line represents the fit of the spectra using Model I (see text for the model details).

the pulse-resolved spectra. The reduced $\chi^{2}$ in all phase bins is very similar for the two models. This does not allow one to choose ultimately between the two spectral functions. The two models will be referred to as Model I (with a "bump") and Model II (with an absorption line at $10 \mathrm{keV}$ ) throughout the paper.

The common part of Models I and II is the power law/cutoff continuum smoothed at the cutoff energy by a third order polynomial:

$$
I_{\text {cont }}=K \cdot \begin{cases}E^{-\Gamma}, & \text { if } E \leq E_{\text {cutoff }}-\Delta E \\ E^{-\Gamma} \cdot \exp \left(-\frac{E-E_{\text {cutoff }}}{E_{\text {fold }}}\right), & \text { if } E>E_{\text {cutoff }}+\Delta E \\ A E^{3}+B E^{2}+C E+D, & \text { if } E_{\text {cutoff }}-\Delta E<E< \\ & <E_{\text {cutoff }}+\Delta E\end{cases}
$$

where $K$ is the normalization coefficient, $E$ is the photon energy; $\Gamma, E_{\text {cutoff }}, E_{\text {fold }}$, and $\Delta E$ are model parameters. Numerical coefficients $A, B, C$, and $D$ are chosen to obey the condition of continuity 

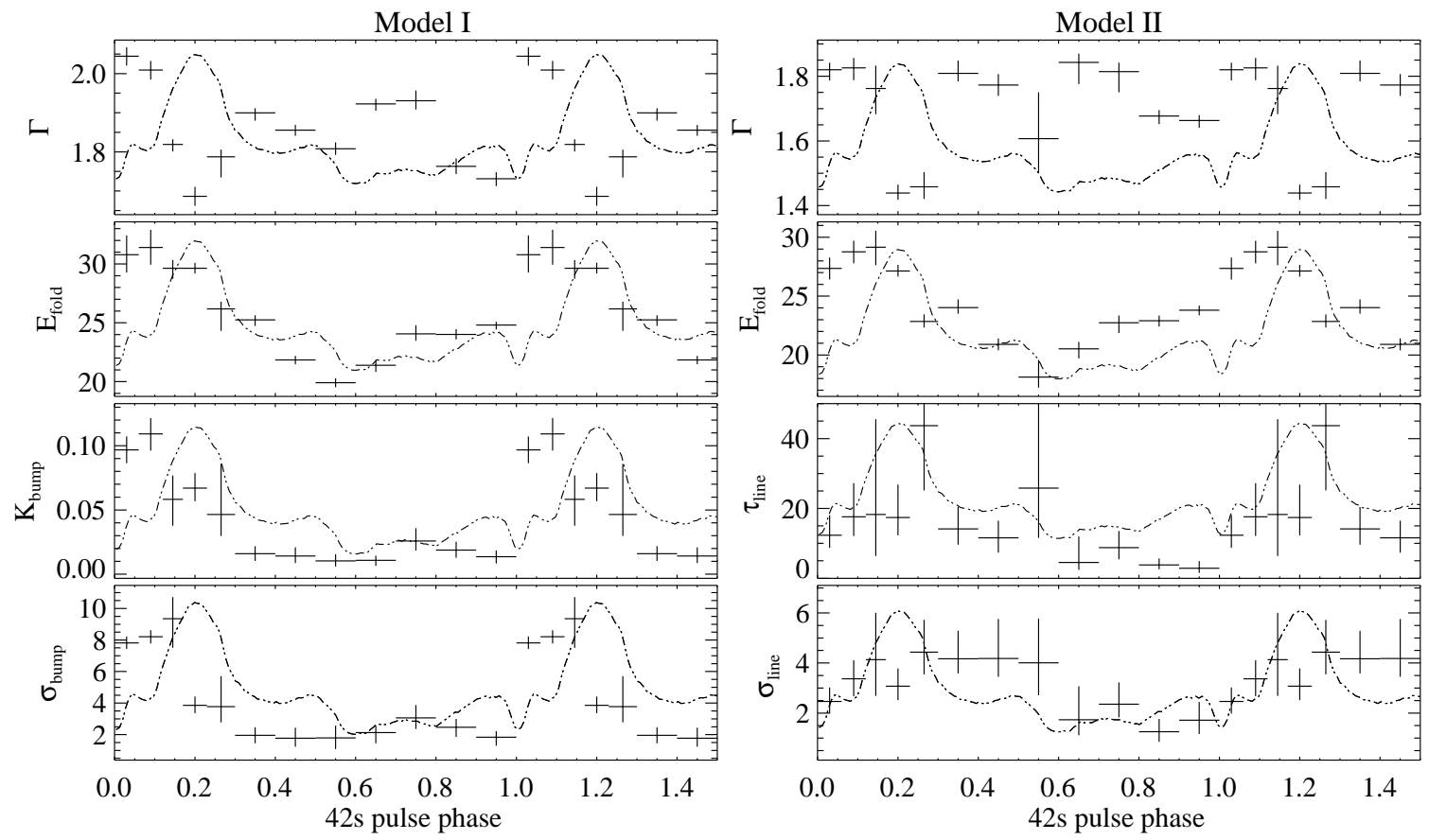

Figure 4: Best fit parameters of Model I (left) and Model II (right) as a function of pulse phase obtained by fitting the spectra from Set 2 . The dotted curve shows the averaged ISGRI (20-120 keV) pulse profile. Vertical error bars correspond to $90 \%$-uncertainties.

for the function and its first derivative at the points $\left(E_{\text {cutoff }}-\Delta E\right)$ and $\left(E_{\text {cutoff }}+\Delta E\right)$. The Model I includes a broad Gaussian emission component (a "bump") around $15 \mathrm{keV}$ :

$$
I_{\text {Model I }}=I_{\text {cont }}+K_{\text {bump }} \exp \left\{-\frac{\left(E-E_{\text {bump }}\right)^{2}}{2 \sigma_{\text {bump }}^{2}}\right\},
$$

where $E_{\text {bump }}$ and $\sigma_{\text {bump }}$ are the energy and width of the "bump". $K_{\text {bump }}$ is the numerical constant describing the intensity of the component. In the Model II the power law/cutoff continuum $I_{\text {cont }}$ is modified by a multiplicative absorption line with a Gaussian optical depth profile:

$$
I_{\text {ModelII }}=I_{\text {cont }} \cdot \exp \left\{-\tau_{\text {line }} \exp \left(-\frac{\left(E-E_{\text {line }}\right)^{2}}{2 \sigma_{\text {line }}^{2}}\right)\right\}
$$

where $E_{\text {line }}, \sigma_{\text {line }}$, and $\tau_{\text {line }}$ are the centroid energy, width, and the central depth of the line, respectively. Additionally, we multiplied the functions $I_{\text {ModelI }}$ and $I_{\text {ModelII }}$ by the factor $\exp \left[-N_{\mathrm{H}} \sigma_{\mathrm{bf}}(E)\right]$ describing the low-energy absorption by cold matter in the line of sight. $\sigma_{\mathrm{bf}}(E)$ is the photoabsorption cross-section per hydrogen atom for matter of cosmic abundances (Bałucińska-Church \& McCammon 1992) used in the phabs model of XSPEC and $N_{\mathrm{H}}$ is the equivalent hydrogen column density. We have also added a Gaussian emission line to model the iron fluorescence line at $\sim 6.4 \mathrm{keV}$. The latter, however, only slightly improves the fit.

Figure 4 shows the most important spectral parameters obtained using the two models as a function of pulse phase. The data correspond to the observations performed in Set 2 which have the best statistics. The same kind of analysis was performed using the observations corresponding 


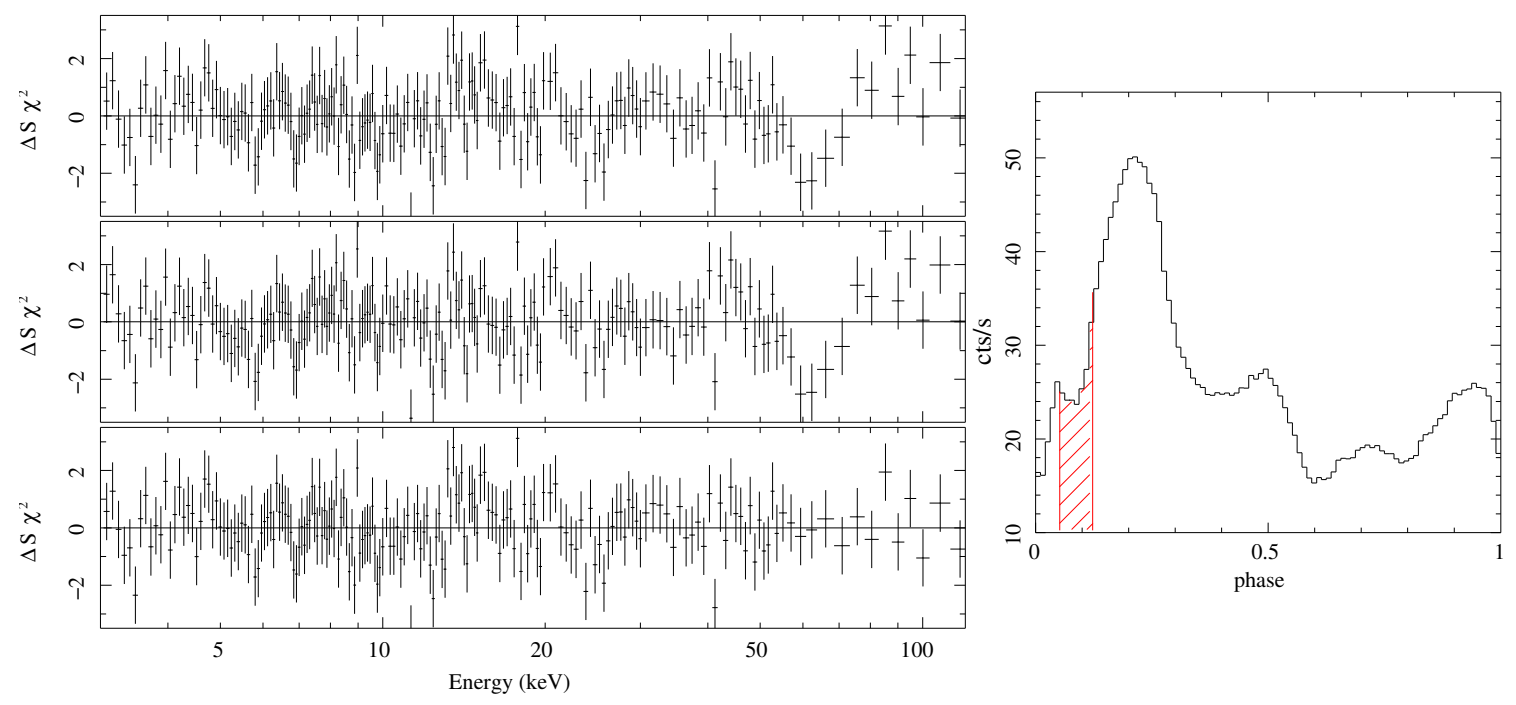

Figure 5: Left: Residuals of the spectrum from the phase bin 0.06-0.12 fitted with Model I (top) and Model II (middle). The bottom panel shows the residuals after fitting the spectrum with Model I where a Gaussian absorption line at $\sim 63.6 \mathrm{keV}$ is included (the corresponding fit with Model II looks very similar). Right: The phase bin $0.06-0.12$ is indicated on the averaged ISGRI pulse profile.

to Set 1 (slightly before the maximum of the outburst). Due to poorer statistics we had to use a coarser binning compared to Set 2 . The spectral parameters of the pulse-resolved spectra were less constrained in this case. However, the behavior of the parameters is in agreement with that found in Set 2.

Residuals left after fitting by Models I and II the spectrum from phase bin 0.06-0.12 obtained in Set 2 reveal an absorption feature at $\sim 63 \mathrm{keV}$ (see Fig. 5). Inclusion of a Gaussian absorption line at this energy flattens the residuals. The best-fit energy of the line is $63.6_{-2.9}^{+3.7} \mathrm{keV}$ for Model I and $63.3_{-2.9}^{+4.0} \mathrm{keV}$ for Model II. We have checked the presence and the energy of the feature using other possible continuum models, such as Fermi-Dirac cutoff (Tanaka 1986) and so-called Negative and Positive power law times EXponential model (NPEX, Makishima et al. 1999), including a "bump" or an absorption line at $10 \mathrm{keV}$ to match the continuum. It was found that the presence and the energy of the feature are independent of the choice of the spectral function. The F-test probability that the line is due to statistical fluctuations is $\sim 3 \times 10^{-5}$ (see however Protassov et al. 2002, about non-applicability of the F-test to line-like features).

\section{Luminosity-dependence of the pulse profile}

As mentioned in Sect. 2, during Set 3 INTEGRAL caught the source at the end of the outburst's decay phase when the X-ray luminosity was $\sim 10$ times lower than that in the maximum of the outburst (Fig 1). Due to lower statistics one cannot perform a detailed pulse-resolved spectral analysis of these data. One can, however, explore the luminosity-dependence of the pulse profile and compare it with that observed with EXOSAT during the 1985 giant outburst.

Figure 6 shows the pulse profiles of the source at the maximum and during the decay of the 2006 giant outburst. The energy range, $2-9 \mathrm{keV}$, approximately corresponds to that of EXOSAT. 


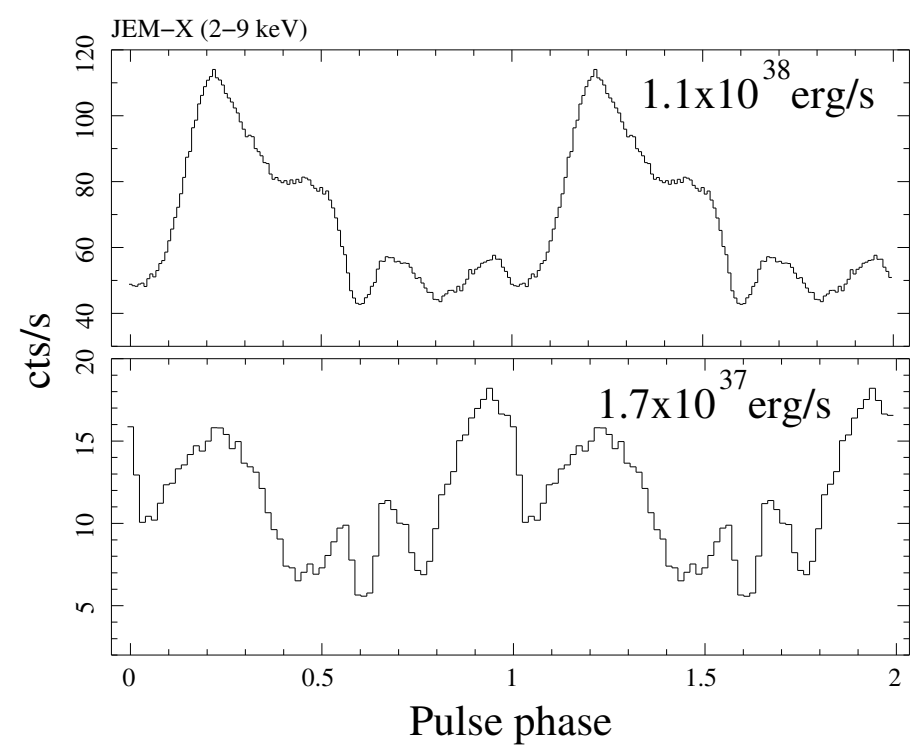

Figure 6: X-ray pulse profile of EXO $2030+375$ obtained with JEM-X in the 2-9 keV energy range during the maximum outburst (top) and at the end of the decay phase (bottom). The indicated luminosity correspods to the distance of $7.1 \mathrm{kpc}$ (Wilson et al. 2002).

The INTEGRAL pulse profiles shown in Fig. 6 are similar to the corresponding EXOSAT profiles from the 1985 giant outburst obtained when the source was at similar luminosity levels (see Fig. 1 in Parmar et al. $1989 a^{2}$ ). This confirms that the shape of the pulse profile of EXO 2030+375 is mainly driven by the X-ray luminosity of the source.

\section{Discussion and conclusions}

\subsection{Variation of the spectral continuum with pulse phase}

The INTEGRAL observations have shown that the spectral continuum of EXO 2030+375 is strongly pulse phase dependent. Observations of the source close to the maximum of the outburst allowed us to perform a detailed study of this dependence. A harder main peak and softer interpulse region observed in the source is a common property of accreting pulsars (see e.g. Tsygankov et al. 2007, and references therein). It is usually explained assuming that during the peak we mostly see the comptonized photons coming from a hot region close to the foot step of the accretion column while in the interpulse softer radiation scattered by the upper parts of the column is observed (Basko \& Sunyaev 1976). A closer look at Fig. 2, however, reveals a more complicated picture. Not only the main peak but also its left flank increases in relative amplitude with energy, leading to a slight shift of the profile maximum towards earlier pulse phase. This shows that the spectral continuum changes asymmetrically with respect to the main peak. The variation of spectral parameters with pulse phase (Fig. 4) demonstrates the corresponding complex pulse phase dependence of the spectrum. The photon index $\Gamma$ reaches a minimum (the hardest spectrum) in the middle of the main

\footnotetext{
${ }^{2}$ The X-ray luminosity of the source reported by (Parmar et al. 1989a) have to be corrected according to the updated distance to the source ( $\sim 7 \mathrm{kpc}$, Wilson et al. 2002).
} 
peak (top panels in Fig. 4) while the maximum of the exponential folding energy $E_{\text {fold }}$ is shifted with respect to the peak towards earlier pulse phases. This is in contrast to e.g. Her X-1 where both the maximum of $E_{\text {fold }}$ and the minimum of $\Gamma$ are coincident with the main pulse (Klochkov et al. 2008).

A possible qualitative explanation for the observed asymmetry of the spectral variation with pulse phase in EXO 2030+375 can be the following. Since the luminosity of the source close to the maximum of the outburst, $\sim 10^{38} \mathrm{erg} \mathrm{s}^{-1}$, is well above the local Eddington limit (so-called critical luminosity, $L_{c} \sim 10^{37} \mathrm{erg} \mathrm{s}^{-1}$, Basko \& Sunyaev 1976; Staubert et al. 2007) it is expected that accreted matter is decelerated by radiation pressure leading to the formation of an optically thick accretion column. The bulk of the emission in this case is expected to occur as a fan beam (see also the modeling of the source's pulse profiles performed by Parmar et al. 1989a). Therefore, during the maximum of the profile (main peak) the angle between the column axis and the observer's line of sight has the largest value (the column is seen from the side). The observer is looking almost along the beam, seeing the photons coming from a Compton scattering region with large optical depth. This leads to the observed hard power law during the peak. Before the peak, when $E_{\text {fold }}$ reaches a maximum, the direction of the line of sight might be closer to that of the column axis and, thus, of the magnetic field lines. Due to the dependence of the scattering cross-section on the angle between the photon direction and the magnetic field lines (Harding \& Daugherty 1991) one expects that the photons, whose direction in this case is closer to that of the field lines, have experienced less scatterings and, therefore, originated deeper inside the accretion column where the temperature is higher. The X-ray spectrum of these photons is expected to have larger $E_{\text {fold }}$ reflecting higher electron temperature but softer power law index due to lower Compton scattering optical depth (see e.g. Rybicki \& Lightman 1979), as observed. This explanation, however, requires that in the latter case the angle between the observer's line of sight and the column axis was not too small. Otherwise, depending on the geometry of the accretion flow, the column density along the line of sight will be very high resulting in a higher optical depth.

The changing of the pulse profile towards the end of the outburst seems to confirm this picture. In the pulse profiles corresponding to the outburst's decay (Fig. 6, bottom panel) one can see a new peak that appears at the phase interval preceeding the main pulse, i.e. where, according to our view, the observer's line of sight is closest to the magnetic field lines. At this pulse phase one expects to see a pencil beam if the luminosity decreases (Reig \& Coe 1998; Parmar et al. 1989a). The observed peak, therefore, may correspond to the pencil beam component of the emission diagram, whose intensity is comaprable to that of the fan beam component at low luminosity levels. So, both the pulse phase variation of the spectral continuum during the maximum of the outburst and the evolution of the pulse profile with luminosity fit to the described picture where the emission diagram changes from a fan beam geometry close to the maximum of the outburst to a combination of a fan and a pencil beam at the end of the decay phase.

\section{2 "Bump" versus absorption line at $10 \mathrm{keV}$}

As discussed in Sect. 4, in order to model the spectral continuum of EXO 2030+375 in the maximum of the outburst one has to modify the standard power law/cutoff model either by a "bump" at $\sim 15 \mathrm{keV}$ or by an absorption line at $\sim 10 \mathrm{keV}$. Both models provide equally good fits of the pulse-phase resolved spectra. If interpreted as a cyclotron absorption feature (Wilson et al. 
2008), the absorption line at $10 \mathrm{keV}$ is observed at rather unusual energy which is much smaller than the exponential cutoff energy $E_{\text {cutoff }}$. This is in contrast to all other know cyclotron line sources (see e.g. Fig. 9 in Coburn et al. 2002). We point out here that a feature around 8-15 keV (a "wiggle" or a "bump") is observed in many accreting pulsars (e.g., 4U 1907, Her X-1, Coburn et al. 2002), including those that do not otherwise exhibit a cyclotron line (e.g. GS 1843+00, Coburn 2001). Thus, the interpretation of the absorption line at $10 \mathrm{keV}$ in EXO 2030+375 as the cyclotron resonant scattering feature might be hasty. On the other hand, the nature of the feature in this and other sources is still unclear. Most probably it arises from modeling the spectral continuum with a simple empirical function. For a proper modeling of the spectrum in the considered energy range (including the feature) one would need a proper theoretical model accounting for all relevant processes at the site of the X-ray emission.

\subsection{Evidence for a cyclotron line at $\sim 63 \mathrm{keV}$}

The X-ray spectrum of EXO 2030+375 taken close to the maximum of the outburst in the narrow pulse phase interval $(0.06-0.12)$ preceeding the main peak shows evidence for an absorption line around $\sim 63 \mathrm{keV}$ (Fig. 5). If interpreted as a fundamental cyclotron line the corresponding magnetic field strength is $B \simeq 5 \times 10^{12} \mathrm{G}\left(E_{\mathrm{cyc}} \sim 11.6 \times\left(B / 10^{12} \mathrm{G}\right) \mathrm{keV}\right)$ which is one of the largest values among accreting pulsars. However, evidence of a cyclotron line at $\sim 36 \mathrm{keV}$ has previously been reported for EXO 2030+375 by Reig \& Coe (1999) during a normal outburst. So, the line at $\sim 63 \mathrm{keV}$ might well be the first harmonic rather than the fundamental line. It is known that the relative strength of the fundamental line and harmonics might vary significantly, sometimes making the fundamental line to be more difficult to detect than the harmonic (a good example is A 0535+26, see e.g. Kendziorra et al. 1994).

\section{References}

Bałucińska-Church, M. \& McCammon, D. 1992, ApJ, 400, 699

Basko, M. M. \& Sunyaev, R. A. 1976, MNRAS, 175, 395

Coburn, W. 2001, PhD thesis, AA(UNIVERSITY OF CALIFORNIA, SAN DIEGO)

Coburn, W., Heindl, W. A., Rothschild, R. E., et al. 2002, ApJ, 580, 394

Coe, M. J. 2000, in ASP Conf. Ser. 214: IAU Colloq. 175: The Be Phenomenon in Early-Type Stars, ed. M. A. Smith, H. F. Henrichs, \& J. Fabregat, 656

Harding, A. K. \& Daugherty, J. K. 1991, ApJ, 374, 687

Kendziorra, E., Kretschmar, P., Pan, H. C., et al. 1994, A\&A, 291, L31

Klochkov, D., Horns, D., Santangelo, A., et al. 2007, A\&A, 464, L45

Klochkov, D., Staubert, R., Postnov, K., et al. 2008, A\&A, 482, 907

Makishima, K., Mihara, T., Nagase, F., \& Tanaka, Y. 1999, ApJ, 525, 978 
Parmar, A. N., White, N. E., \& Stella, L. 1989a, ApJ, 338, 373

Parmar, A. N., White, N. E., Stella, L., Izzo, C., \& Ferri, P. 1989b, ApJ, 338, 359

Protassov, R., van Dyk, D. A., Connors, A., Kashyap, V. L., \& Siemiginowska, A. 2002, ApJ, 571, 545

Reig, P. \& Coe, M. J. 1998, MNRAS, 294, 118

Reig, P. \& Coe, M. J. 1999, MNRAS, 302, 700

Rybicki, G. B. \& Lightman, A. P. 1979, Radiative processes in astrophysics (New York, WileyInterscience, 1979. 393 p.)

Staubert, R., Shakura, N. I., Postnov, K., et al. 2007, A\&A, 465, L25

Tanaka, Y. 1986, in Lecture Notes in Physics, Berlin Springer Verlag, Vol. 255, IAU Colloq. 89: Radiation Hydrodynamics in Stars and Compact Objects, ed. D. Mihalas \& K.-H. A. Winkler, 198

Tsygankov, S. S., Lutovinov, A. A., Churazov, E. M., \& Sunyaev, R. A. 2007, ArXiv e-prints, 704

Wilson, C. A., Finger, M. H., \& Camero-Arranz, A. 2008, ApJ, 678, 1263

Wilson, C. A., Finger, M. H., Coe, M. J., Laycock, S., \& Fabregat, J. 2002, ApJ, 570, 287

Winkler, C., Courvoisier, T. J.-L., Di Cocco, G., et al. 2003, A\&A, 411, L1

Ziolkowski, J. 2002, Memorie della Societa Astronomica Italiana, 73, 1038 